\title{
Directional Emission and Universal Far-Field Behavior from Semiconductor Lasers with Limacon-Shaped Microcavity
}

\section{Citation}

Yan, Changling, Qi Jie Wang, Laurent Diehl, Martina Hentschel, Jan Wiersig, Nanfang Yu, Christian Pflügl et al. 2009. Directional emission and universal far-field behavior from semiconductor lasers with limacon-shaped microcavity. Applied Physics Letters 94(25): 251101.

\section{Published Version}

http://dx.doi.org/10.1063/1.3153276

\section{Permanent link}

http://nrs.harvard.edu/urn-3:HUL.InstRepos:5096753

\section{Terms of Use}

This article was downloaded from Harvard University's DASH repository, and is made available under the terms and conditions applicable to Other Posted Material, as set forth at http:// nrs.harvard.edu/urn-3:HUL.InstRepos:dash.current.terms-of-use\#LAA

\section{Share Your Story}

The Harvard community has made this article openly available.

Please share how this access benefits you. Submit a story.

\section{Accessibility}




\title{
Directional emission and universal far-field behavior from semiconductor lasers with limaçon-shaped microcavity
}

\author{
Changling Yan, ${ }^{1,2}$ Qi Jie Wang, ${ }^{1, a)}$ Laurent Diehl, ${ }^{1}$ Martina Hentschel, ${ }^{3}$ Jan Wiersig, ${ }^{4}$ \\ Nanfang Yu, ${ }^{1}$ Christian Pflügl, ${ }^{1}$ Federico Capasso, ${ }^{1, b)}$ Mikhail A. Belkin, ${ }^{5}$ \\ Tadataka Edamura, ${ }^{6}$ Masamichi Yamanishi, ${ }^{6}$ and Hirofumi Kan ${ }^{6}$ \\ ${ }^{1}$ School of Engineering and Applied Sciences, Harvard University, Cambridge, Massachusetts 02138, USA \\ ${ }^{2}$ Changchun University of Science and Technology, Changchun 130022, People's Republic of China \\ ${ }^{3}$ Max-Planck-Institut für Physik Komplexer Systeme, Nöthnitzer Straße 38, D-01187 Dresden, Germany \\ ${ }^{4}$ Institut für Theoretische Physik, Universität Magdeburg, Postfach 4120, D-39016 Magdeburg, Germany \\ ${ }^{5}$ Department of Electrical and Computer Engineering, University of Texas at Austin, \\ Texas 78758, USA \\ ${ }^{6}$ Central Research Laboratories, Hamamatsu Photonics K. K., Shizuoka 434-8601, Japan
}

(Received 2 April 2009; accepted 10 May 2009; published online 22 June 2009)

\begin{abstract}
We report experimental demonstration of directional light emission from limaçon-shaped microcavity semiconductor lasers. Quantum cascade lasers (QCLs) emitting at $\lambda \approx 10 \mu \mathrm{m}$ are used as a model system. Both ray optics and wave simulations show that for deformations in the range $0.37<\varepsilon<0.43$, these microcavities support high quality-factor whispering gallerylike modes while having a directional far-field profile with a beam divergence $\theta_{\|} \approx 30^{\circ}$ in the plane of the cavity. The measured far-field profiles are in good agreement with simulations. While the measured spectra show a transition from whispering gallerylike modes to a more complex mode structure at higher pumping currents, the far field is insensitive to the pumping current demonstrating the predicted "universal far-field behavior" of this class of chaotic resonators. Due to their relatively high quality factor, our microcavity lasers display reduced threshold current densities compared to conventional ridge lasers with millimeter-long cavities. The performance of the limaçon-shaped QCLs is robust with respect to variations of the deformation near its optimum value of $\varepsilon=0.40$. (C) 2009 American Institute of Physics. [DOI: 10.1063/1.3153276]
\end{abstract}

Microcavity lasers have attracted a lot of attention in recent years due to the simplicity of their fabrication and low threshold currents, which makes them suitable for highdensity optoelectronic integration. ${ }^{1}$ Microdisk lasers have low threshold current densities but their optical power output is very low due to total internal reflection of the whispering gallery modes (WGMs) and their far-field profiles are isotropic. Such properties limit their potential applications.

To overcome the intrinsic problems of microdisk lasers, various types of deformed structures ${ }^{2-4}$ were proposed, and/or demonstrated in a variety of gain media including polymers and semiconductors. Among these, electrically pumped semiconductor microcavity lasers such as stadiumshaped lasers, ${ }^{5}$ bow-tie lasers, ${ }^{6}$ and spiral-shaped lasers ${ }^{7-9}$ are of special interests for potential applications and as tool to study ray and wave chaos. Up to now, however, very few studies of microcavities in both experiment ${ }^{10}$ and theory ${ }^{11,12}$ have shown promise of achieving directional emission while having high quality-factor ( $Q$-factor) modes in the cavity.

Recently, a limaçon-shaped microcavity has been proposed $^{13}$ as a promising resonator shape for microcavity lasers with attractive properties such as a directional emission and a high cavity $Q$-factor. In this work, we fabricated $\lambda \approx 10 \mu \mathrm{m}$ quantum cascade lasers (QCLs) with limaçonshaped microcavity and characterized their performance. We observed directional emission from our devices with a farfield divergence angle $\theta_{\|} \approx 33^{\circ}$ in the plane of the cavity and

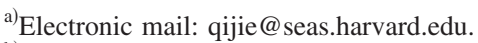

${ }^{\text {b)} E l e c t r o n i c ~ m a i l: ~ c a p a s s o @ ~ s e a s . h a r v a r d . e d u . ~}$
}

a $Q$-factor of more than 1000 at the midinfrared wavelength. The measured far-field profiles of our devices are in excellent agreement with simulations.

The boundary of a limaçon microcavity is defined in polar coordinate as $R(\theta)=R_{0}(1+\varepsilon \cos \theta)$ where $\varepsilon$ is the deformation factor and $R_{0}$ is the radius of curvature when $\theta$ $=\pi / 2$ [see the inset of Fig. 1(a)]. In this work, we first carried out wave simulations based on the boundary element method $^{13}$ to study the effect of the parameter $\varepsilon$ on the key characteristics of the limaçon microcavity QCLs such as $Q$-factor and directionality of the light emission. Note that the polarization of QCL is transverse magnetic (TM) due the selection rules of the optical transition. The effective refractive index of our QCL material (lattice matched $\mathrm{Ga}_{0.47} \mathrm{In}_{0.53} \mathrm{As} / \mathrm{Al}_{0.48} \mathrm{In}_{0.52} \mathrm{As} / \mathrm{InP}$ ) for TM polarization, $n$, is estimated to be 3.2, calculated from spectral measurement of the mode spacing of a Fabry-Pérot type ridge laser. Figures 1 (a) -1 (c) show the intensity distribution of some TM modes calculated for a structure with $\varepsilon=0.40$ and $R_{0}=80 \mu \mathrm{m}$. The two highest $Q$-factor modes are shown, respectively, in Figs. 1(a) and 1(b); they both have a calculated $Q$-factor $>10^{7}$, assuming no material loss in the laser cavity. Since these WGMs have the highest $Q$-factor, they can be excited at pumping currents just above the lasing threshold. The mode in Fig. 1(c) is a non-WGM which has a lower $Q$-factor of about 18000 and as such we expect this type of mode will be excited at higher pumping currents. Figure 1(d) shows the Poincaré surface of section (SOS) of rays leaving the limaçon cavity with $\varepsilon=0.4$ and $R_{0}=80 \mu \mathrm{m}$ calculated with ray optics simulations (see Ref. 13 for details). The character 


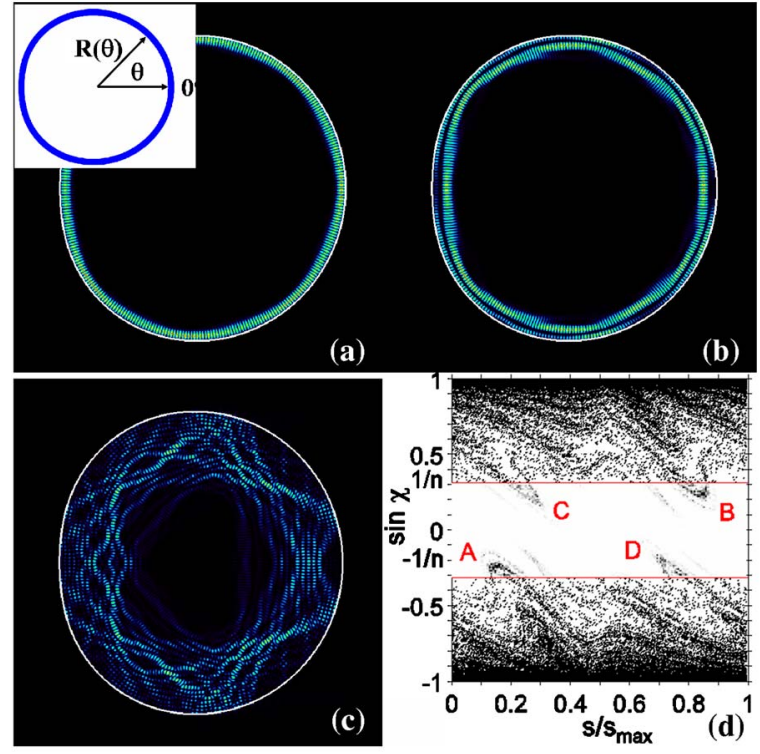

FIG. 1. (Color online) Intensity distribution of the TM modes inside the cavity calculated with wave simulations $\left(\varepsilon=0.40\right.$ and $\left.R_{0}=80 \mu \mathrm{m}\right)$; (a) The highest $Q$-factor WGM; the inset shows the schematic structure of the limaçon microcavity with $\varepsilon=0.40$; (b) The second highest $Q$-factor WGM; (c) One of the low $Q$-factor modes; (d) Poincaré SOS of rays leaving the Limacon cavity where $s$ (measured from the $\theta=0$ direction with $s_{\max }$ being the cavity circumference) is the arclength and $\chi$ is the angle of incidence at each reflection at the cavity boundary. 30000 rays are started along the cavity boundary with WG-like initial conditions $(0.8<|\sin \chi|<1)$ and followed until they cross the critical lines $|\sin \chi|=1 / n$ where they start to escape from the cavity. Between the critical lines, the ray intensity is weighted by the Fresnel coefficients (for TM polarization). The resulting accumulated intensity is shown in gray scale and represents the so-called unstable manifold which determines the far-field pattern of the cavity.

of the ray trajectories is chaotic, while the far-field profile is determined by the path in phase space that the rays take to escape the cavity by entering the leaky region, where the condition of total internal reflection is not fulfilled anymore.

Both wave and ray optics simulations show in good agreement that the deformation $\varepsilon=0.40$ results in the smallest far-field divergence angle of about $30^{\circ}$ [defined as the full width at half maximum (FWHM) of the main far-field lobe around $\theta=0$ line], as shown in Fig. 2(a). The inset of Fig. 2(a) shows the external intensity distribution of the mode in Fig. 1(a) obtained by wave simulation. The main peaks are labeled as $\mathrm{A}^{\prime}, \mathrm{B}^{\prime}, \mathrm{C}^{\prime}$, and $\mathrm{D}^{\prime}$ corresponding to the escape regions $\mathrm{A}, \mathrm{B}, \mathrm{C}$, and $\mathrm{D}$, respectively, as marked in Fig. 1(d). Interestingly, we observed [Fig. 2(b)] that all three modes in Figs. 1(a)-1(c) show similar external far-field profiles no matter whether they are high $Q$-factor WGMs or low
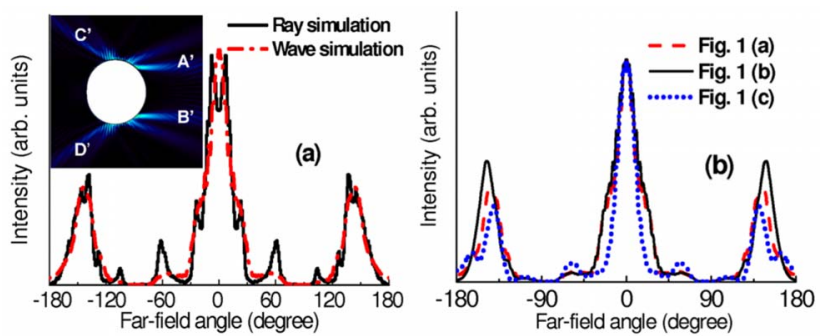

FIG. 2. (Color online) (a) Comparison of the far-field profiles obtained with wave simulation [corresponding to the optical mode in Fig. 1(a)] and ray optics simulation; the inset is the external intensity distribution of the mode in Fig. 1(a); (b) Wave simulations of the far-field profiles of the three modes in Figs. 1(a)-1(c). All profiles are normalized to their maximum values.

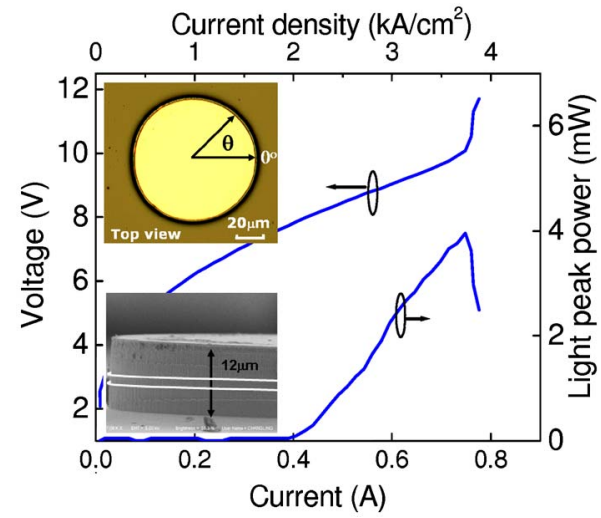

FIG. 3. (Color online) Voltage and peak output power as a function of injection current for the limaçon-shaped microcavity QCL with a deformation factor $\varepsilon=0.40$ and $R_{0}=80 \mu \mathrm{m}$; the upper left inset is the top view of the device taken with an optical microscope; and the lower left inset is the scanning electron microscope image of the side view of the device; the two white lines indicate the position of the active region.

$Q$-factor non-WGMs. This is what is called "universal farfield behavior," which was also observed previously in quadrupole deformed microcavities. ${ }^{14}$ The reason for this universal far-field behavior is that the emission directionality is mainly determined by the structure of the unstable manifolds in the leaky region, as shown in Fig. 1(d), which are determined by the geometric shape of the deformed microcavity, regardless of the different spatial distributions of these modes inside the cavity.

The QCL material used was similar to the one described in Ref. 15 but with a different doping level ( $\sim 30 \%$ lower) in the active region. Devices with different sizes $R_{0}=50,80$, and $110 \mu \mathrm{m}$ and deformations $\varepsilon$ ranging from 0.20 to 0.80 were fabricated. Inductively coupled plasma reactive ion etching was used to etch the QCL material. The top view and the side view of a typical device are shown in the inset of Fig. 3. The sidewall roughness is about $300 \mathrm{~nm}$, which is expected to result only in minor scattering of the midinfrared radiation.

The processed devices were tested in pulsed mode at room temperature with $125 \mathrm{~ns}$ current pulses at $80 \mathrm{kHz}$ repetition rate. All devices demonstrated laser action. Figure 3 shows the light output power versus current $(L-I)$ and voltage versus current $(V-I)$ characteristics of a representative device with $\varepsilon=0.40$ and $R_{0}=80 \mu \mathrm{m}$. Peak output power of 4 $\mathrm{mW}$, a threshold current density around $2.0 \mathrm{kA} / \mathrm{cm}^{2}$, and a maximum slope efficiency of about $12 \mathrm{~mW} / \mathrm{A}$ were obtained. This device has a smaller threshold current density compared with that $\left(\sim 2.6 \mathrm{kA} / \mathrm{cm}^{2}\right)$ of ridge QCLs with a length of $2.5 \mathrm{~mm}$ and $14 \mu \mathrm{m}$ width processed from the same wafer. The slope efficiency of the device is lower than that $(\sim 100 \mathrm{~mW} / \mathrm{A})$ of the ridge QCLs because not all of the pumping area of the limaçon microcavity is utilized for the output optical power generation (see Fig. 1) due to the presence of WGMs in the cavity. For this device, a $Q$-factor of approximately 1200 was obtained based on the measurements of the threshold current density and the gain coefficient. ${ }^{16}$ Although the measured $Q$-factor is smaller than those of shorter wavelength semiconductor lasers ${ }^{17}$ as a result of increase in waveguide losses, it is larger than the $Q$-factors reported for other circular-shaped QCLs emitting at similar wavelengths. ${ }^{16,18}$ This is assigned to material and device processing improvements and to the limaçon resona- 

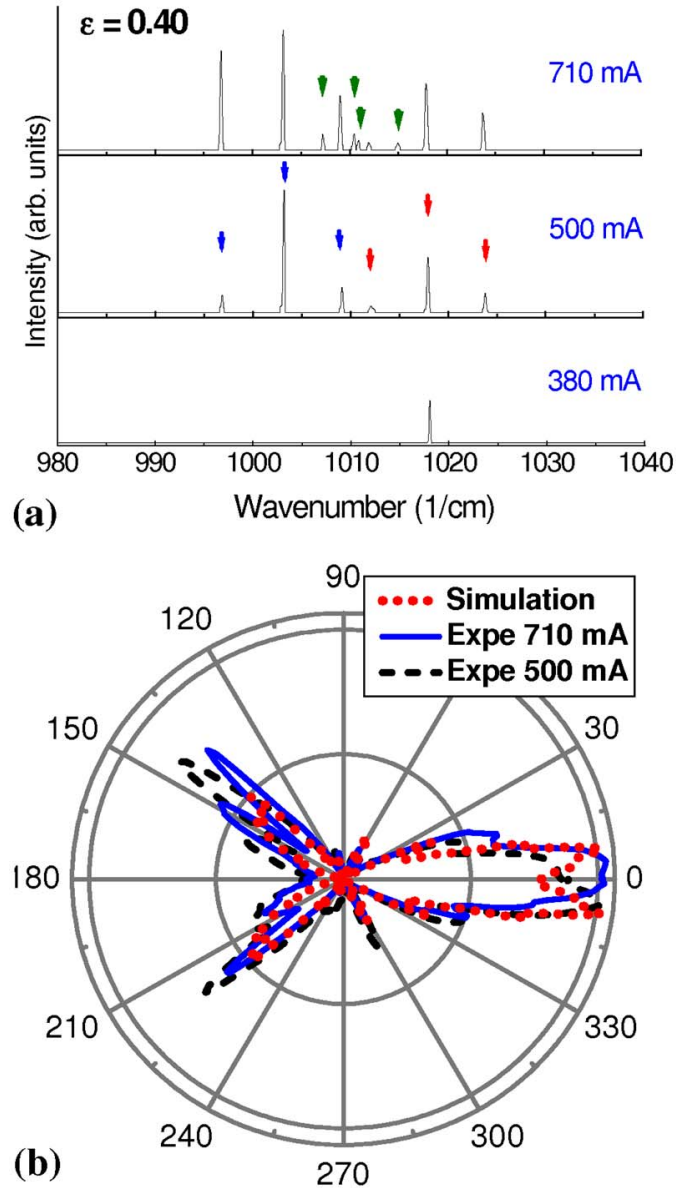

FIG. 4. (Color online) Experimental results for a limaçon microcavity QCL with $\varepsilon=0.40$ and $R_{0}=80 \mu \mathrm{m}$. (a) Laser spectra at different pumping currents. The threshold current of the laser is around $380 \mathrm{~mA}$; at $500 \mathrm{~mA}$ pumping current, two sets of WGMs are shown, expected to correspond to the two set modes in Figs. 1(a) and 1(b); at a higher pumping current (710 mA), several non-WGMs appear; (b) Comparison between ray optics simulation and experimental lateral far-field profiles in polar coordinates at pumping currents of 500 and $710 \mathrm{~mA}$. All far-field profiles are normalized to their maximum values.

tor, which supports high $Q$-factor WGMs. We note that, due to the high optical losses associated with free carrier absorption at midinfrared wavelength, the measured $Q$-factor in our devices is much smaller than the value obtained in simulations.

Figure 4(a) shows the emission spectra of the limaçon microcavity QCL measured at different pumping currents along the $\theta=0$ direction with a high-resolution Fourier transform infrared spectrometer. The laser operates in single mode at $\lambda \approx 10 \mu \mathrm{m}$ at the threshold current of $380 \mathrm{~mA}$. At a pumping current of $500 \mathrm{~mA}$, two sets of optical modes appeared, indicated by red and blue arrows. It is reasonble to assume that they correspond to the two high $Q$-factor WGMs shown in Figs. 1(a) and 1(b), respectively. The average mode spacing of each set is approximately $6.0 \mathrm{~cm}^{-1}$, which agrees very well with the calculated value $\left(6.2 \mathrm{~cm}^{-1}\right)$ for WGMs, given by $1 /\left(L^{*} n\right)$, where $L$ is the perimeter of the structure. At higher pumping current, several additional unequally spaced modes appeared, indicated by green arrows, corresponding to lower $Q$-factor modes (non-WGMs) of the type shown in Fig. 1(c). We also observed essentially the same spectra from all far-field lobes in different directions.
The far-field profiles of our devices were measured in steps of $0.5^{\circ}$ using a setup described in Ref. 15. The experimental results for a device with an optimal (in terms of the far-field divergence angle) deformation $\varepsilon=0.40$ are shown in Fig. 4(b) for pumping currents of 500 and $710 \mathrm{~mA}$, together with the ray optics simulation. Excellent agreement is achieved between experiment and simulation. Note that although non-WGMs appear at higher pumping current of 710 $\mathrm{mA}$ [see Fig. 4(a)], the far-field profile is essentially the same as the one pumped at $500 \mathrm{~mA}$ showing directional emission due to the universal far-field behavior predicted for this type of resonator. The measured FWHM of the main lobe of the far-field profile is $\sim 33^{\circ}$. The measured divergence is also similar to the one reported for rational caustic resonator ${ }^{10}$ $\left(\sim 35^{\circ}\right)$ and that of Fabry-Pérot type ridge laser $\left(\sim 40^{\circ}\right) .{ }^{15}$

Very good agreement between the calculated and the measured far-field profiles was also observed with $\varepsilon$ different from 0.4 . We note, however, for $\varepsilon$ larger than 0.5 , the geometry of the microcavity is such that WGMs are not supported anymore in the cavity. The device performance is also insensitive to the deformation in the range of $0.37<\varepsilon<0.43$ well within the fabrication resolution of photolithography for $R_{0}$ $=80 \mu \mathrm{m}$ devices.

This work was supported by the AFOSR (Grant No. FA9550-08-1-0047). Financial support from the DFG research group 760 is gratefully acknowledged by Jan Wiersig and Martina Hentschel who in addition acknowledges support within the DFG Emmy-Noether Programme. The structures were processed in the Center for Nanoscale Science (CNS) in Harvard University. Harvard-CNS is a member of the National Nanotechnology Infrastructure Network. Changling Yan acknowledges support from the China Scholarship Council (CSC) visiting scholarship.

${ }^{1}$ K. J. Vahala, Nature (London) 424, 839 (2003).

${ }^{2}$ A. F. J. Levi, R. E. Slusher, S. L. McCall, J. L. Glass, S. J. Pearton, and R. A. Logan, Appl. Phys. Lett. 62, 561 (1993).

${ }^{3}$ Optical Processes in Microcavities, edited by R. K. Chang and A. J. Campillo (World Scientific, New York, 1996).

${ }^{4}$ J. U. Nöckel and A. D. Stone, Nature (London) 385, 45 (1997).

${ }^{5}$ W. Fang, H. Cao, and G. S. Solomon, Appl. Phys. Lett. 90, 081108 (2007).

${ }^{6}$ C. Gmachl, F. Capasso, E. E. Narimanov, J. U. Nöckel, A. D. Stone, J. Faist, D. L. Sivco, and A. Y. Cho, Science 280, 1556 (1998).

${ }^{7}$ G. D. Chern, H. E. Tureci, A. D. Stone, R. K. Chang, M. Kneissl, and N. M. Johnson, Appl. Phys. Lett. 83, 1710 (2003).

${ }^{8}$ R. Audet, M. A. Belkin, J. A. Fan, B. G. Lee, K. Lin, F. Capasso, E. E. Narimanov, D. Bour, S. Corzine, J. Zhu, and G. Höfler, Appl. Phys. Lett. 91, 131106 (2007).

${ }^{9}$ D. X. Qu, R. Cendejas, Z. J. Liu, C. Gmachl, and F. Towner, Appl. Phys. Lett. 93, 261116 (2008).

${ }^{10}$ Y. Baryshnikov, P. Heider, W. Parz, and V. Zharnitsky, Phys. Rev. Lett. 93, 133902 (2004).

${ }^{11}$ J. Wiersig and M. Hentschel, Phys. Rev. A 73, 031802(R) (2006).

${ }^{12}$ M. Hentschel and T.-Y. Kwon, Opt. Lett. 34, 163 (2009).

${ }^{13}$ J. Wiersig and M. Hentschel, Phys. Rev. Lett. 100, 033901 (2008).

${ }^{14}$ S.-B. Lee, J. Yang, S. Moon, J.-H. Lee, K. An, J.-B. Shim, H.-W. Lee, and S. W. Kim, Phys. Rev. A 75, 011802(R) (2007).

${ }^{15}$ N. Yu, J. Fan, Q. J. Wang, C. Pflügl, L. Diehl, T. Edamura, M. Yamanishi, H. Kan, and F. Capasso, Nat. Photonics 2, 564 (2008).

${ }^{16}$ C. Gmachl, J. Faist, F. Capasso, C. Sirtori, D. L. Sivco, and A. Y. Cho, IEEE J. Quantum Electron. 33, 1567 (1997).

${ }^{17}$ J. Faist, C. Gmachl, M. Striccoli, C. Sirtori, F. Capasso, D. L. Sivco, and A. Y. Cho, Appl. Phys. Lett. 69, 2456 (1996).

${ }^{18}$ S. Gianordoli, L. Hvozdara, G. Strasser, W. Schrenk, K. Unterrainer, and E. Gornik, Appl. Phys. Lett. 75, 1045 (1999). 